\title{
Erratum: Funding crunch forces stem cell company to abandon therapies
}

Monya Baker

Nat. Biotechnol. 25, 951-952, 2007; published online 3 September 2007

In the version of this article originally published, in the penultimate paragraph, the name of the Monash University scientist was misspelled; the correct spelling is Colin Pouton. In addition, the statement in paragraph 2 "the SSCC, which is being supported by the same group that funded ESI, the Agency for Science, Technology and Research (A*STAR)” is incorrect. ESI is not funded by A*STAR. Its major investor is Bio-One Capital, a venture capital fund controlled through the Singapore Government's Economic Development Board. The SSCC is funded by A*STAR.

\section{Erratum: Mice with a human touch}

\section{Christopher Thomas Scott}

Nat. Biotechnol. 25, 1075-1077, 2007; published online 6 October 2007

In the version of this article initially published, it was incorrectly stated on p. 1076, paragraph 2, line four from bottom, that "Aya Jakobovits, originally at Cell Genesys and later at Abgenix, shepherded the XenoMouse through its entire development." Five of the six XenoMouse strains developed at Abgenix were initiated after Jakobovits left in 1997.

\section{Erratum: Discovery and development of the complement inhibitor eculizumab for the treatment of paroxysmal nocturnal hemoglobinuria}

\author{
Russell P Rother, Scott A Rollins, Christopher F Mojcik, Robert A Brodsky \& Leonard Bell \\ Nat. Biotechnol. 25, 1256-1264 (2007); published online 7 November 2007; corrected after print 7 December 2007
}

In the version of this article initially published, on p. 1258, paragraph 2, the incorrect serum concentrations to reach steady state and the incorrect accumulation ratio $\left(\mathrm{R}_{\mathrm{acc}}\right)$ for eculizumab were given. The corrected sentence reads "Eculizumab serum concentrations appear to reach steady state after $\sim 57$ days. At steady state, the eculizumab accumulation ratio $\left(\mathrm{R}_{\mathrm{acc}}\right)$ was calculated to be 1.075." In addition, in Figure 5, in the PDF version only, the word "assessment" was cut off the bottom of the box beginning "September 2006." The final sentence should read "BLA and MAA submitted to FDA and EMEA, respectively; receive accelerated assessment." And in Table 1, the heading "Units of packed RBCs transfused/patient/month" now appears in a separate row. The errors have been corrected in the HTML and PDF versions of the article.

\section{Corrigendum: A diverse family of thermostable cytochrome P450s created} by recombination of stabilizing fragments

Yougen Li, D Allan Drummond, Andrew M Sawayama, Christopher D Snow, Jesse D Bloom \& Frances H Arnold Nat. Biotechnol. 25, 1051-1056 (2007); published online 26 August 2007; corrected after print 7 December 2007

In the version of this article initially published, the sentence starting with "The most thermostable 450 P450 chimera" contains an extra " 450 ." The correct sentence should start as follows "The most thermostable P450 chimera". The error has been corrected in the HTML and PDF versions of the article. 